\title{
Using social media for HIV/Aids Communication
}

\author{
Jagadeeswaran $\mathrm{M}^{1 *}$, Vikashini. $\mathrm{S}^{2}$, Saravanan $\mathrm{R}^{3}$, Haripriya. $\mathrm{M}^{4}$ \\ ${ }^{1}$ Professor, Department of Pharmaceutical Analysis, Nandha College of Pharmacy, \\ Erode, Tamilnadu, Email:jaganmurugesh@gmail.com \\ ${ }^{2}$ Assistant Professor, Department of Pharmacy Practice, Nandha College of Pharmacy, \\ Erode, Tamilnadu, Email: vikashini@nandhapharmacy.org \\ ${ }^{3}$ Associate Professor, Department of Pharmacology, Nandha College of Pharmacy, \\ Erode, Tamilnadu, Email: saravanan@nandhapharmacy.org \\ ${ }^{4}$ Assistant Professor, Department of Child Health Nursing, Nandha College of Nursing, \\ Erode, Tamilnadu, Email: karthipriyavibi@gmail.com \\ ${ }^{*}$ Corresponding Author
}

\begin{abstract}
Social media assumes a significant part in regular viewpoint and it is indistinguishable in all methods for exercises. This paper means to recognize which credits of web-based media add to the medium being seen as either advantageous or restricting when used as a mode for conveying HIV/AIDS-data to online multitudes and gatherings. To more readily comprehend the work of online media in HIV/AIDS correspondence, web-based media's application as a correspondence vehicle for general wellbeing data. This is done because of the way that exploration examining the sending of web-based media for wellbeing purposes as an expansive going term, is more pervasive than for HIV/AIDS correspondence explicitly.
\end{abstract}

Keywords: social media, HIV, Communication

\section{Introduction}

The rise of innovation interceded correspondence stages, for example, web-based media, have been instrumental in the difference in intuition as they offer the chance of scattering custom fitted wellbeing correspondence messages to firmly characterized gatherings. Nonetheless, the effect of web-based media with regards to HIV/AIDS explicit correspondence, and the resulting difficulties for correspondence technique advancement, show up still generally neglected. As the connection among business and innovation for wellbeing is quickly creating, web-based media's utility for wellbeing correspondence is getting more obvious. Numerous examinations have zeroed in on the Internet as a correspondence channel to narrowcast and tailor messages inside wellbeing correspondence. With broad communications regularly worried about contacting general crowds, the Internet, as a correspondence medium, has empowered the conveyance of intuitive and media wellbeing related substance to explicitly characterized gatherings. These components, among others, are vital for the making of the necessary sensation of connectedness among clients. To highlight understudies' impression of the medium utilized in HIV/AIDS correspondence for this exploration, these and different variables will be looked into to survey whether it adds to, or upsets compelling correspondence.
Inspected the viability of Internet intercessions, particularly its dispersal potential. Discoveries show that customized informing and interpersonal interaction usefulness may build the take-up of Internet intercession content. Shutting holes in plan and coordinating usefulness was featured for its capability to add to dispersal of data. Checked on techniques and procedures for Internet-conveyed wellbeing conduct change intercessions focused on teenagers or youthful grown-ups. Examples of powerful techniques highlighted customized correspondence, the utilization of updates, and motivating forces. As wellbeing data Internet clients apparently see substance and local area as the most relevant applications for wellbeing related correspondence, advertisers needing to create correspondence systems, should hence fixate their consideration on these applications. With regards to this examination, these two applications are viewed as the pertinent web-based media utilities. Utilizing web-based media for data chasing (substance) or utilizing online media to cooperate (local area), are additionally investigated.

\section{E-health}

At the start, it is basic to see how individuals utilize the Internet for health related exercises. This could help health correspondence advertisers to plan correspondence procedures that would be with regards to use designs which may pull in and hold 
clients and improve information. E-health arose as a trendy expression, trying to diagram the joined utilization of electronic correspondence and data innovation for general comfort. Advertisers purportedly first utilized the word with an end goal to impart the prospects of internet business in the medical services area. The principle objective of ehealth is to improve the strength of individuals through the ideal utilization of data and correspondence innovation. The joined use of innovation intervened stages with wellbeing, can create, catch, send, store, and recover advanced information for different purposes.

E-health opens up the information base of wellbeing data and medication to buyers and their organized local area. Welfare administrations are reached out past the customary limits - both geographic and reasonable - as purchasers are currently ready to acquire wellbeing administrations through innovation interceded online applications, from suppliers across the globe. E-patients, or people who are prepared, empowered, enabled and occupied with their wellbeing and medical services choices are expanding in numbers and expect a wellbeing customer experience like what might be offered to them in the business domain

The distinguished arising e-wellbeing patterns may add to medical care through online media, by expanding admittance to medical care, Fostering a conversation on innovation and business in the medical services domain, filling in as an intersection to encourage input among customers and business for the improvement of medical care and helping medical care offices, for example, clinics, to broaden their scope by methods for innovation.

\section{Social media for health communication}

Having logically been utilized for the spread of wellbeing messages as of late are of the view that web-based media have been appeared to support commitment, grow reach, and encourage admittance to trustworthy wellbeing data. Despite the fact that clients progressively recognize assembling and sharing wellbeing data through web-based media stages, they additionally yield being mindful while doing so conceding that its application as a hotspot for wellbeing data likewise has potential entanglements which ought not to be overlooked. Social media clients are searching out and devouring health data utilizing web-based media. Observational investigations show a wealth of casual and coordinated wellbeing related correspondence previously occurring on driving web-based media stages. Besides, the capability of online media to affect wellbeing conduct through improved information, coming about because of admittance to data, has itself gotten expanding consideration of late. Most of flow research is centered on how organizations can use web-based media to pull in new purchasers, generally for business acquire. There is a requirement for research zeroing in on how online media can be utilized to spread health explicit data to different crowds. As in most current advertising methodologies, while utilizing online media as a component of an incorporated health explicit promoting interchanges procedure, advertisers should look to advance from absolutely dispersing wellbeing related data, to drawing in with the intended interest group. The correspondence should plan to encourage local area support, rouse the acknowledgment of new sound conduct, and make a stage where clients can share individual encounters. Further, web-based media can help advertisers of wellbeing related messages to contact individuals when, where and how they like to get the correspondence. As demonstrated in the above conversation, the social setting of searching out data identifying with a person's very own wellbeing or the soundness of those in one's very own organization, individual crowds request dynamic investment and sharing of data. More than the actual message, drawing in clients to co-make content, to rate, rank and remark on interchanges, is seen as elevating the realness of messages, improving trust in, and building clients' associations with an element . When creating on the web innovation intervened health correspondence systems, it is fundamental to do an investigation of the target group's inclinations and necessities. Various examinations likewise underline the meaning old enough in wellbeing correspondence endeavors using online media, stressing that range and effect will be ideal when a more youthful age is focused on. By sharing activity situated examination, advertisers of wellbeing correspondence messages can encourage a superior comprehension on how best to bridle the capability of online media as a correspondence mechanism for a particular general wellbeing result. This accepts considerably more noteworthy significance, explicitly in a HIV/AIDS setting, where issues of shame and protection are still profoundly pertinent.

\section{Social media for HIV/AIDS communication}

Existing examination via social media's sending as a correspondence platform for HIV/AIDS data predominantly centers on current events and difficulties for communicators, its capability to arrive at youths and the moral contemplations connected. The conceivable usage or adequacy of online media as a correspondence mechanism for HIV/AIDS-related data among need and high-hazard gatherings. Effect of online media in this specific situation and the ensuing difficulties for correspondence methodology advancement. By consistently observing new advancements in creating markets such health correspondence can assess its effect for future rebusiness. To abuse the potential, advance a superior arrangement, and distinguish the most practical accessible methodologies, which can be arranged related to the qualities of the different web-based media stages, essential examination is fundamental .Further, the information on understudies' web-based 
media utilization may likewise give a system that empowers advertisers to more readily comprehend what inspires innovation intervened social support for health advancement when all is said in done. . Correspondence channel to improve information and bring issues to light with respect to HIV/AIDS explicitly has delivered clashing outcomes. One perspective is that expanded online access has made it simpler to disperse data at one's own accommodation. A restricting perspective is that online media in this setting may yield unintended negative effects, for example, the dispersal of nontenable wrong data. As this is a quickly evolving field, the apparent advantages and constraints ought not to be viewed as a thorough rundown but instead as characteristic of the premium that it draws from scholarly analysts and advertisers the same.

The models were taken to survey issues identifying with HIV counteraction research among youth on interpersonal interaction locales on the web and adequacy of the utilization of web-based media for general wellbeing data correspondence. To be considered advantageous, the recognized web-based media credits needed to cling to the accompanying above rules

\section{Conclusion}

Concluding up from the examination, innovation intervened stages benefit advertisers the chance to impart health related data to customers. With the expanding event of e-health and the developing number of buyers searching out health data on the web, web-based media makes a state of conjunction, which justifies its utilization for health related correspondence. Health specialist co-ops are besides given a chance to team up with buyers, and consequently give items or administrations that would seemingly improve the strength of their objective business sectors. Along these lines, buyers are empowered to more readily direct their own health in return, by having the option to get to wellbeing data in an innovation intervened climate with which they are as of now recognizable. Further, in measuring discernments it is imperative to consider that different elements may impact one's insight. Along these lines, different online media characteristics may also impact discernment in a great or negative way. To evaluate whether the recognized ascribes can be considered as either helpful or restricting to viable correspondence, these credits themselves require further investigation. This additionally thus requires that the part of the correspondence channel itself be assessed. The section that follows audits speculations and models of both a correspondence channel and the issue of discernment, and moreover gives a short outline of the common hypotheses utilized for HIV/AIDS correspondence procedure advancement.

\section{References}

1. Hughes D, Rowe M, Batey M, Lee A. A tale of two sites: Twitter vs Facebook and the personality predictors of social media usage. Computers in Human Behavior. 2012 Mar;28(2):561-569.

2. Coursaris CK, Liu M. An analysis of social support exchanges in online HIV/AIDS self-help groups. Computers in Human Behavior. 2009 Jul;25(4):911-918.

3. L. Yonker, et al. "Friending teens: a systematic review of social media in adolescent and young adult health care. (J Med Internet Res. PubMed: 25560751)

4. Ventola CL. Social Media and Health Care Professionals: Benefits, Risks, and Best Practices. Pharm Ther. 2014; 39:491-520.

5. Rosenmann, A., and Safir, M.P. 2007. Gay identity nonendorsement and the role of dissociative characteristics in a culturally diverse online sample of men who have sex with men. Sexuality Research \& Social Policy 4:18-26.

6. Van de Ven, P., Mao, L., Fogarty, A., Rawstorne, P., Crawford, J., Prestage, G., Grulich, A., Kaldor, J., and Kippax, S. 2005. Undetectable viral load is associated with sexual risk taking in HIV serodiscordant gay couples in Sydney. AIDS 19:179-184.

7. Saunders, M., Lewis, P. \& Thornhill, A. 2007. Research Methods for Business Students. 4th ed. Essex: Prentice Hall.

8. Rimal, R. N. \& Adkins, A. D. 2003. Using computers to narrowcast health messages: The role of audience segmentation, targeting, and tailoring in health promotion. (In Thompson, T. L., Dorsey, A. M., Miller, K. I. \& Parrott, R. Handbook of Health Communication. Mahwah, NJ: L. Erlbaum Associates. p.497-515.)

9. Pudliner, B. A. 2007. Alternative literature and tourist experience: Travel and tourist weblogs. Journal of Tourism and Cultural Change, 5(1): 46-59.

10. Mccarthy, J. 2011. "Social mediator": Bridging the gaps between $\mathrm{HCl}$ and social media. Interactions, March \& April, 15-18. 